\title{
Efeito de diferentes modalidades de treinamento físico no consumo de oxigênio de pico em pacientes pós-infarto agudo do miocárdio: uma revisão sistemática e metanálise
}

\author{
Effect of different physical training modalities on peak oxygen consumptions in \\ post-acute myocardial infarction patients: systematic review and meta-analysis
}

Gabriela Bourscheid ${ }^{1}$ (D), Karin Raquel Just ${ }^{1}$, Rochelle Rocha Costa ${ }^{2,3}$ (D), Thalia Petry ${ }^{4}$, Luiz Cláudio Danzmann ${ }^{5}$ (D), Adamastor Humberto Pereira², (D), Alexandre Araújo Pereira, ${ }^{2,6}$, Leandro Tolfo Franzoni 2,6 (D),

Eduardo Lima Garcia ${ }^{6}$ (1D)

\begin{abstract}
Resumo
O treinamento físico é capaz de aumentar o consumo de oxigênio de pico em indivíduos que sofreram infarto agudo do miocárdio. No entanto, em relação à eficácia de diferentes tipos de intervenções ainda há uma lacuna na literatura. O objetivo do presente estudo foi avaliar os efeitos de diferentes modalidades de treinamento físico no consumo de oxigênio de pico de pacientes pós-infarto agudo do miocárdio. Foram utilizadas as seguintes bases de dados: PubMed (MEDLINE), Cochrane Library, Scopus e PEDro. Foram incluídos estudos que avaliassem exercícios aeróbicos, de força ou combinados. Seis estudos preencheram elegibilidade. O exercício aeróbico aumentou $6,07 \mathrm{~mL} . \mathrm{kg}^{-1} \cdot \mathrm{min}^{-1}$ quando comparado ao grupo controle $(p=0,013)$. Na comparação entre exercício combinado e grupo controle, foi observada uma diferença de 1,84 $\mathrm{mL} \cdot \mathrm{kg}^{-1} \cdot \mathrm{min}^{-1}$, no entanto, sem significância ( $p=0,312$ ). Portanto, concluímos que o exercício aeróbico é a única modalidade que apresenta eficácia para aumentar o consumo de oxigênio de pico em comparação a um grupo controle.
\end{abstract}

Palavras-chave: exercício físico; infarto agudo do miocárdio; reabilitação cardiovascular; doença da artéria coronariana; cardiopatia isquêmica.

\begin{abstract}
Physical training can increase peak oxygen uptake (VO2peak) in people who have suffered acute myocardial infarction (AMI). However, there is still a gap in the literature in relation to the effectiveness of different types of interventions. Therefore, the aim of the present study was to evaluate the effects of different physical training modalities on VO2peak in post-AMI patients. The following databases were used: PubMed (MEDLINE), Cochrane Library, Scopus, and PEDro. Studies that evaluated aerobic exercise, strength exercise, or combined exercise were included. Six studies met eligibility criteria. Aerobic exercise increased VO2peak by $6.07 \mathrm{ml} . \mathrm{kg}^{-1} \cdot \mathrm{min}^{-1}$ when compared to the control group (CG) $(p=0.013)$. The comparison between combined exercise and control group detected a difference of $1.84 \mathrm{ml}_{\mathrm{kg}}^{-1}$. $\mathrm{min}^{-1}$, but this was not significant $(\mathrm{p}=0.312$ ). We therefore conclude that aerobic exercise is the only modality that is effective for increasing VO2peak compared to a control group.
\end{abstract}

Keywords: physical exercise; acute myocardial infarction; cardiovascular rehabilitation; coronary artery disease; ischemic cardiopathy.

Como citar: Bourscheid G, Just KR, Costa RR, et al. Efeito de diferentes modalidades de treinamento físico no consumo de oxigênio de pico em pacientes pós-infarto agudo do miocárdio: uma revisão sistemática e metanálise. J Vasc Bras. 2021;20: e20210056. https://doi.org/10.1590/1677-5449.210056

1 Faculdade Cenecista de Santo Ângelo, Santo Ângelo, RS, Brasil.

${ }^{2}$ Universidade Federal do Rio Grande do Sul - UFRGS, Porto Alegre, RS, Brasil.

${ }^{3}$ Faculdade SOGIPA, Porto Alegre, RS, Brasil.

${ }^{4}$ Universidade Federal de Santa Maria - UFSM, Santa Maria, RS, Brasil.

${ }^{5}$ Universidade Luterana do Brasil - ULBRA, Canoas, RS, Brasil.

${ }^{6}$ Hospital de Clínicas de Porto Alegre - HCPA/UFRGS, Porto Alegre, RS, Brasil.

Fonte de financiamento: Nenhuma.

Conflito de interesse: Os autores declararam não haver conflitos de interesse que precisam ser informados.

Submetido em: Abril 08, 2021. Aceito em: Maio 08, 2021.

O estudo foi realizado na Faculdade Cenecista Santo Ângelo, vinculado à Universidade Federal do Rio Grande do Sul (UFRGS), Porto Alegre, RS, Brasil.

Copyright $(2021$ Os autores. Este é um artigo publicado em acesso aberto (Open Access) sob a licença Creative Commons Attribution, que permite uso, distribuição e reprodução em qualquer meio, sem restrições desde que o trabalho original seja corretamente citado. 


\section{INTRODUÇÃO}

A mortalidade por doenças cardiovasculares (DCVs) representa cerca de $70 \%$ das mortes no mundo, chegando a mais de 38 milhões de mortes por ano ${ }^{1}$. No Brasil, a taxa de mortalidade por DCVs chega a $30 \%{ }^{2}$. Entre 2008 e 2016, o Sistema Único de Saúde (SUS) brasileiro efetuou 2.548 .944 procedimentos envolvendo doenças isquêmicas do coração, variando de tratamento clínico para infarto agudo do miocárdio (IAM) até cirurgia de revascularização do miocárdio ${ }^{3}$. Em 2018, o SUS gastou cerca de R\$ 3.700.000,00 com procedimentos envolvendo isquemia miocárdica ${ }^{3}$. Estima-se que a mortalidade por IAM em 2017 foi de $56 \%{ }^{4}$.

O IAM está francamente relacionado à carga de doença aterosclerótica ${ }^{5}$, mas sobretudo vinculado à aguda formação trombótica oclusiva sobre uma placa ateromatosa coronariana, a qual gera oclusão da luz do vaso e necrose miocárdica ${ }^{6,7}$. O grau de irreversibilidade da aterosclerose está diretamente associado a lesões avançadas, como, por exemplo, fibroateromas ${ }^{8}$. A necrose formada por um núcleo rico em lipídeos é ocasionada pela degradação da matriz extracelular, morte de células musculares lisas e apoptose das células espumosas, promovendo acúmulo de lipídeos ${ }^{9}$. Por fim, os fibroateromas causam calcificação arterial, compondo parte da placa de oclusão, definida pela presença de um trombo arterial ${ }^{10}$.

A disfunção miocárdica pós-infarto é o fator preponderante à redução da capacidade funcional do paciente. Alterações na capacidade de contratilidade do músculo cardíaco impossibilitam a elevação da frequência cardíaca e pressão arterial em baixas cargas de esforço físico, reduzem o duplo produto e geram um limiar isquêmico baixo. $\mathrm{O}$ teste cardiopulmonar de exercício é considerado padrão-ouro para avaliação da capacidade funcional, por meio do consumo de oxigênio de pico (VO2pico) ${ }^{11,12}$. Diferentes estudos demonstram que o VO2pico é um preditor independente de mortalidade para indivíduos que sofreram $\mathrm{IAM}^{13-}$ ${ }^{15}$. Além disso, um aumento de $1 \mathrm{~mL} \cdot \mathrm{kg}^{-1} \cdot \mathrm{min}^{-1}$ no VO2pico está diretamente associado a uma redução de $10 \%$ no risco de mortalidade por DCVs ${ }^{16,17}$.

Entre as diferentes formas não medicamentosas para tratar o IAM, o exercício físico é uma ferramenta de extrema importância, tanto na prevenção dos fatores de risco como no aumento do $\mathrm{VO} 2 \mathrm{pico}^{18}$. O exercício aeróbico é o mais recomendado nas atuais diretrizes de reabilitação cardíaca ${ }^{19-21}$, pois é o mais simples de ser realizado fora do ambiente hospitalar, sem necessidade de equipamentos ${ }^{22}$. Sobre a eficácia, o exercício aeróbico parece apresentar resultados semelhantes em relação ao VO2pico comparado a outros modelos, como, por exemplo, exercício combinado (aeróbico + força na mesma sessão) ${ }^{23}$, e ser superior a exercício de força ${ }^{24}$. No entanto, existe uma lacuna na literatura em relação à comparação da eficácia de diferentes tipos de treinamento físico sobre o VO2pico em indivíduos que sofreram IAM. Diante disso, o objetivo do presente estudo foi conduzir uma metanálise, comparando os efeitos de diferentes tipos de treinamento físico sobre o VO2pico de indivíduos que sofreram IAM.

\section{MÉTODOS}

\section{Caracterização da pesquisa}

O estudo foi caracterizado como revisão sistemática com metanálise. Foram seguidas as recomendações propostas pela Cochrane Collaboration ${ }^{25}$ e pelo Preferred Reporting Items for Systematic Review and Meta-analyses (PRISMA) Statement ${ }^{26}$. A presente revisão foi previamente registrada no International Prospective Register of Systematic Reviews (PROSPERO) (CRD42020182666).

\section{Critérios de elegibilidade}

Foram incluídos ensaios clínicos randomizados, que avaliaram o efeito do exercício físico sobre VO2pico em indivíduos que sofreram IAM, sem restrições de idade e sexo. Além disso, foram incluídos estudos que submetessem os indivíduos à cirurgia de revascularização do miocárdio/endovascular ou tratamento conservador com medicamento antitrombótico para reverter o quadro de isquemia.

Os modelos de exercício físico foram restritos a três tipos: exercício aeróbico, exercício de força e exercício combinado, sem restrição em relação ao tipo de exercício, instrumentos, intensidade, duração da sessão, frequência semanal, volume ou intervalo de descanso. A duração mínima da intervenção foi fixada em 6 semanas, entendida como tempo mínimo necessário para o efeito positivo no VO2pico em pacientes após IAM, e sua realização deveria ter acontecido de modo supervisionado.

Além disso, os estudos deveriam apresentar comparações dos resultados com grupos de indivíduos que não realizaram exercício (grupo controle) ou realizaram o exercício preconizado pelas diretrizes (grupo controle padrão pela literatura - exercício aeróbico de moderada intensidade). Apenas foram incluídos estudos que não relataram diferença significativa entre os grupos antes do início do período de seguimento. Não houve restrições quanto à data de publicação para inclusão dos estudos na revisão, e estudos publicados em inglês e português foram incluídos na análise. 


\section{Critérios de exclusão}

Foram excluídos da presente revisão sistemática estudos que comparassem técnicas cirúrgicas com técnicas não cirúrgicas. Além disso, foram excluídos estudos observacionais ou estudos que não apresentassem dados suficientes para a extração dos dados.

\section{PROCEDIMENTOS PARA COLETA DE DADOS}

\section{Estratégia de busca}

Os artigos inicialmente passaram por um filtro realizado com o software EndNote, para excluirmos as duplicatas. Após, foram analisados títulos e resumos, com base nos critérios de elegibilidade, por dois avaliadores independentes e com experiência. Depois da seleção dos artigos, foram analisados na íntegra aqueles que preencheram os critérios de elegibilidade. Não foi utilizado filtro para data de publicação, visando abranger maior gama de estudos.

Utilizamos os seguintes bancos de dados eletrônicos: PubMed (MEDLINE - US National Library of Medicine), Cochrane Library, Scopus e PEDro (Physiotherapy Evidence Database). Além disso, foi realizada uma busca manual nas referências dos estudos incluídos na pesquisa. Não foi utilizado filtro para ensaios clínicos randomizados, visto que durante a fase inicial optamos por incluir mais estudos para ampliar a abrangência de literatura sobre o assunto. A seleção em relação ao delineamento do estudo foi realizada de maneira manual. Resumos ou resumos expandidos publicados em conferências, dissertações, teses ou estudos ainda não publicados em periódicos (pre-print) não foram incluídos.

A pergunta PICO foi desenvolvida com a finalidade de realizar a busca e orientar a estratégia de seleção dos estudos:

População: pacientes que apresentem infarto agudo do miocárdio;

Intervenção: exercício físico (aeróbico, força ou combinado);

Comparadores: grupo controle, placebo ou exercício aeróbico;

Outcomes (desfechos): consumo de oxigênio de pico.

A estratégia de busca utilizada no PubMed foi:

Population: "Myocardial Infarction"[Mesh] $O R$ "Infarction, Myocardial" OR "Infarctions, Myocardial” OR "Myocardial Infarctions" OR "Cardiovascular Stroke” OR "Cardiovascular Strokes" OR "Stroke, Cardiovascular" OR "Strokes, Cardiovascular" OR "Myocardial Infarct" $O R$ "Infarct, Myocardial" OR "Infarcts, Myocardial" OR "Myocardial Infarcts" OR "Heart Attack" OR "Heart Attacks"
Intervention: "Exercise"[Mesh] OR "Exercises" OR "Physical Activity" OR "Activities, Physical" OR "Activity, Physical" OR "Physical Activities" OR "Exercise, Physical" OR "Exercises, Physical" $O R$ "Physical Exercise" OR "Physical Exercises" OR "Acute Exercise" OR "Acute Exercises" OR "Exercise, Acute" OR "Exercises, Acute” OR "Exercise, Isometric" OR "Exercises, Isometric" OR "Isometric Exercises" OR "Isometric Exercise" OR "Exercise, Aerobic" OR "Aerobic Exercise" OR "Aerobic Exercises" OR "Exercises, Aerobic" $O R$ "Exercise Training" OR "Exercise Trainings" $O R$ "Training, Exercise" OR "Trainings, Exercise" OR "Resistance Training”"[Mesh] OR "Training, Resistance" OR "Strength Training” OR "Training, Strength" OR "Weight-Lifting Strengthening Program" OR "Strengthening Program, WeightLifting” OR "Strengthening Programs, WeightLifting” OR "Weight Lifting Strengthening Program" OR "Weight-Lifting Strengthening Programs" OR

"Weight-Lifting Exercise Program" OR "Exercise Program, Weight-Lifting” OR "Exercise Programs, Weight-Lifting” OR "Weight Lifting Exercise Program" OR "Weight-Lifting Exercise Programs" OR "Weight-Bearing Strengthening Program" $O R$ "Weight-Bearing Strengthening Program"

OR "Strengthening Program, Weight-Bearing"

OR "Strengthening Programs, Weight-Bearing"

OR "Weight Bearing Strengthening Program" OR

"Weight-Bearing Strengthening Programs" $O R$

"Weight-Bearing Exercise Program" OR "Exercise Program, Weight-Bearing” OR "Exercise Programs, Weight-Bearing” OR "Weight Bearing Exercise

Program" OR "Weight-Bearing Exercise Programs”.

Optamos em não utilizar os desfechos na estratégia de busca com a finalidade de incluir uma ampla a literatura sobre o assunto central, incluindo apenas população e intervenção.

Nas demais bases de dados utilizamos apenas os termos MeSH "Myocardial Infarction", "Exercise" e "Resistance Training", visto que para Cochrane Library, PEDro e SciELO não é necessário inserir todos outros termos.

\section{SELEÇÃO DOS ESTUDOS E EXTRAÇÃO DOS DADOS}

Dois revisores independentes (GB e KRJ) avaliaram títulos e resumos de todos os artigos encontrados por meio da estratégia de busca. Posteriormente, foi feita a leitura completa dos artigos selecionados e daqueles sobre os quais se tinha dúvida pelos mesmos dois revisores independentes, seguindo os critérios que determinam inclusão e exclusão dos estudos. As discordâncias entre os dois avaliadores foram resolvidas por consenso. No caso de discordância ou de dúvida, 
um terceiro avaliador (LTF) estava disponível para decidir pela inclusão ou exclusão do estudo.

Os mesmos revisores independentes realizaram a extração dos dados. Foi criado um formulário padronizado, indicando quais informações deveriam ser selecionadas, incluindo, por exemplo, dados de caracterização da amostra, informações clínicas relevantes, como tempo de IAM, medicamentos utilizados e a descrição completa e detalhada das intervenções realizadas. Os dados extraídos foram: ano do estudo, tamanho amostral, sexo, número de homens, número de mulheres, média de idade, desvio padrão da idade, massa corporal média, desvio padrão da massa corporal, índice de massa corporal (IMC) médio, desvio padrão do IMC, característica do grupo, tempo de IAM, tipo de cirurgia, medicamentos, tipo de treinamento, frequência semanal, duração do seguimento, progressão, séries, repetições, intensidade, intervalo e volume. Além disso, foram extraídos os dados do desfecho principal do estudo, VO2pico (média e desvio padrão do período pré e pós-intervenção).

\section{AVALIAÇÃO DA QUALIDADE METODOLÓGICA (RISCO DE VIÉS)}

Para dados relacionados à extração da qualidade metodológica seguimos as orientações da Cochrane Collaboration $^{25}$, incluindo: geração da sequência aleatória (randomização), sigilo da alocação, cegamento do paciente e terapeuta, cegamento dos avaliadores dos desfechos, descrição de perdas e exclusões e dados de resultados incompletos. Dois revisores independentes participaram dessa fase de avaliação (ELG e LTF) para cada critério, e os estudos foram classificados como alto risco (caso não apresentasse o critério), baixo risco (caso apresentasse o critério) e risco não claro (caso o critério não fosse reportado).

\section{ANÁLISE DOS DADOS}

Os resultados são apresentados como diferença média padronizada para valores absolutos entre as intervenções com intervalo de confiança de $95 \%$ (IC95\%). Heterogeneidade estatística dos efeitos das intervenções entre os estudos foi avaliada pelo teste $\mathrm{Q}$ de Cochran e teste de inconsistência $I^{2}$, sendo que valores acima $50 \%$ indicam alta heterogeneidade ${ }^{27}$. O modelo de efeitos aleatórios foi aplicado. A metanálise compreendeu valores de comparação de VO2pico, expressos em $\mathrm{mL} \cdot \mathrm{kg}^{-1} \cdot \mathrm{min}^{-1}$, entre exercício aeróbico versus controle e exercício combinado versus controle. Valores de $\alpha \leq 0,05$ foram considerados estatisticamente significativos. Todas as análises foram realizadas usando o software Comprehensive Meta-Analysis versão 2.0 (Englewood, New Jersey, USA).

\section{RESULTADOS}

Dos 4.586 estudos identificados, oito preencheram os critérios de inclusão (Figura 1). Entretanto, os dados de VO2pico de um estudo estavam na unidade de medida L. min $^{-1}$ e outro não foi randomizado ${ }^{28,29}$. Após contato com os autores, eles informaram que não havia dados em $\mathrm{mL} \cdot \mathrm{kg}^{-1} \cdot \mathrm{min}^{-1}$, unidade de medida

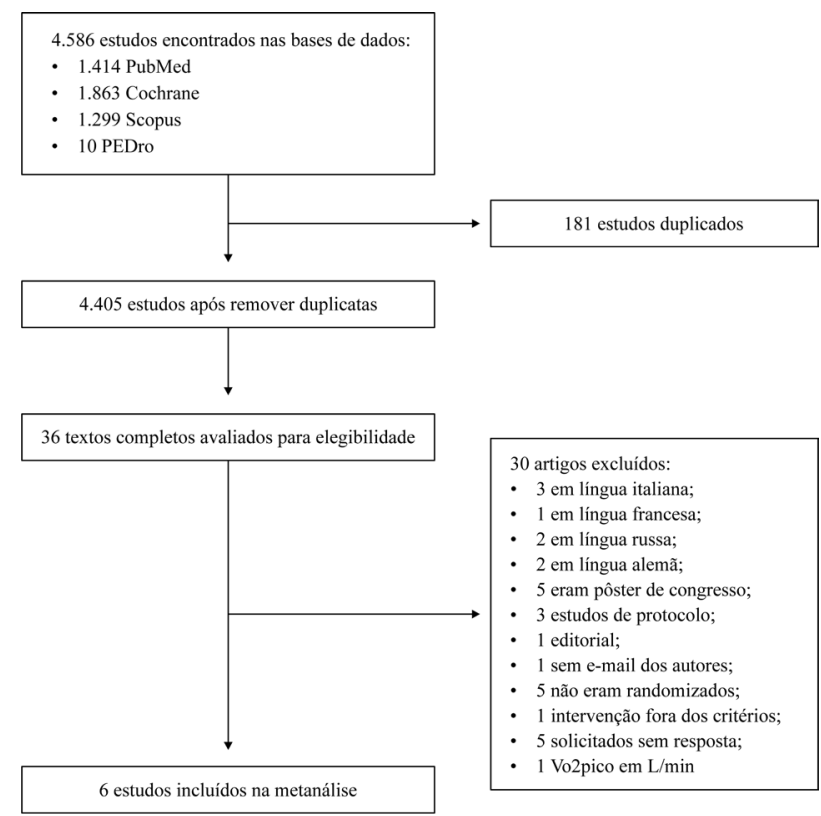

Figura 1. Fluxograma do estudo com todas etapas da revisão sistemática e metanálise. 
padronizada para expressar essa variável. Portanto, seis estudos foram incluídos na análise quantitativa ${ }^{30-35}$. Desses, dois foram incluídos duplamente ${ }^{30,32}$, visto que preencheram os critérios de elegibilidade para duas comparações entre grupos: exercício aeróbico de moderada intensidade versus controle e exercício aeróbico de alta intensidade versus controle. Além disso, dois estudos preencheram os critérios de elegibilidade para comparação entre exercício combinado versus controle (treinamento aeróbico).

No total, 361 participantes foram incluídos na metanálise. Desses, 155 foram incluídos no grupo exercício aeróbico (GEA), 35 no grupo exercício combinado (GEC) e 171 no grupo controle (GC). Cinquenta por cento dos estudos analisaram apenas pessoas do sexo masculino $30,33,34$, sendo que $33 \%$ analisaram pessoas de ambos os sexos e apenas um estudo $(17 \%)$ não descreveu o sexo da amostra incluída. O número total de mulheres foi de 35 (9,7\%).

Dois estudos (33\%) apresentaram amostra composta por indivíduos sedentários e com histórico de obesidade ${ }^{30,33}$. Além disso, $50 \%$ apresentaram fatores de risco, como diabetes mellitus, hipertensão arterial sistêmica, dislipidemia e histórico de tabagismo ${ }^{31,33-35}$. Exercício aeróbico foi a modalidade prevalente entre os seis estudos $(66 \%)$, sendo que, entre os quatro estudos de exercício aeróbico, dois foram adicionados duplamente, em função de apresentarem diferentes intensidades de exercício. A moderada intensidade foi utilizada em três desses quatro estudos $(75 \%)^{30,32,33}$, e alta intensidade também em três estudos $(75 \%)^{30,32,35}$. O exercício combinado foi utilizado em dois estudos $(34 \%)$, sendo que os dois utilizaram alta intensidade para o exercício aeróbico durante o exercício combinado ${ }^{31,34}$

O tempo médio de duração dos programas de intervenção foi de 10,33 $\pm 2,66$ semanas, sendo que a frequência semanal teve duração média de $3,17 \pm 0,98$ e o tempo médio de duração das sessões foi de 45,83 $\pm 9,52$ minutos. Dos seis estudos incluídos, $66 \%$ apresentaram o tempo médio após os participantes terem sofrido o IAM ( $22 \pm 21$ semanas). A Tabela 1 mostra as principais características dos estudos incluídos. A Tabela 2 traz os aspectos relacionados ao risco de viés.

\section{EFEITOS DAS INTERVENÇÕES}

\section{Exercício Aeróbico vs. Controle (com e sem intervenção)}

Um total de 290 participantes foram avaliados (Figura 2), 155 para o GEA e 135 para o GC. O exercício aeróbico foi associado a um aumento médio de $6,07 \mathrm{~mL} \cdot \mathrm{kg}^{-1} \cdot \mathrm{min}^{-1}\left(\right.$ IC95\% 1,27 a 10,86; $\left.I^{2}: 88 \%\right)$ quando comparado ao $\mathrm{GC}(\mathrm{p}=0,013)$.

\section{Exercício Combinado vs. Controle (sem intervenção)}

Setenta e um participantes foram avaliados (Figura 3). Desses, 35 eram do GEC e 36 eram do GC. O exercício combinado não apresentou diferença estatisticamente significativa quando comparado ao GC. No entanto, apresentou aumento médio de $1,84 \mathrm{~mL} \cdot \mathrm{kg}^{-1} \cdot \mathrm{min}^{-1}\left(\mathrm{IC} 95 \%-1,73\right.$ a 5,$\left.42 ; I^{2}: 0 \%\right)$ quando comparado ao $\mathrm{GC}(\mathrm{p}=0,312)$.

\section{DISCUSSÃO}

O presente estudo conduziu uma metanálise para investigar os efeitos de diferentes tipos de treinamento físico sobre o VO2pico de indivíduos que sofreram IAM. Seu principal resultado é que o exercício aeróbico foi associado a um aumento médio de $6 \mathrm{~mL} \cdot \mathrm{kg}^{-1} \cdot \mathrm{min}^{-1}$ no VO2pico em comparação a um GC, com diferença estatisticamente significativa $(\mathrm{p}=0,013)$.

\section{Estudos}

Benetti et al. A 2010

Benetti et al. B 2010 Moholdt et al. A 2011 Oliveira et al. A 2014 Santi et al. A 2018 Santi et al. B 2018

Estimativa (IC95\%)
$\begin{array}{rrr}14.700 & (12.074, & 17.326) \\ 8.300 & (5.141, & 11.459) \\ 2.100 & (-2.457, & 6.657) \\ 2.200 & (-2.019, & 6.419) \\ 3.800 & (-2.332, & 9.932) \\ 3.900 & (-1.448, & 9.248)\end{array}$

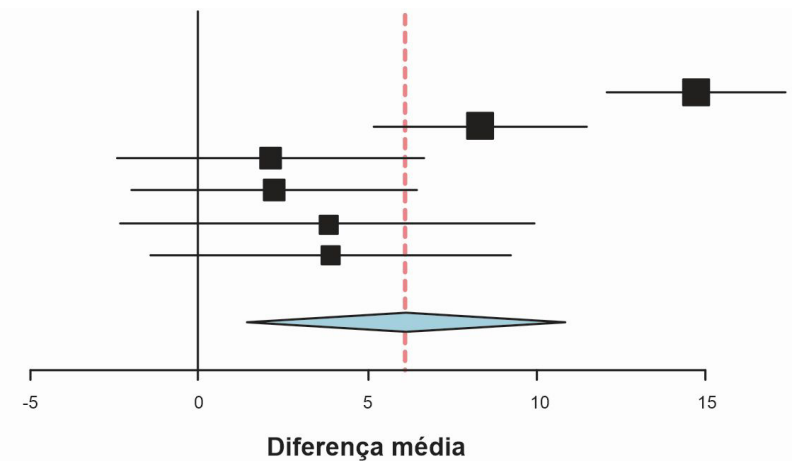

Figura 2. Diferenças médias padronizadas no consumo de oxigênio de pico $\left(\mathrm{mL} \cdot \mathrm{kg}^{-1} \cdot \mathrm{min}^{-1}\right)$ observadas entre exercício aeróbico e grupo controle.

Nota: Quadrado preenchido = estimativa dos estudos específicos; Diamante preenchido = estimativas combinadas de metanálises de efeitos aleatórios; Diferença padronizada; Intervalo de confiança. 


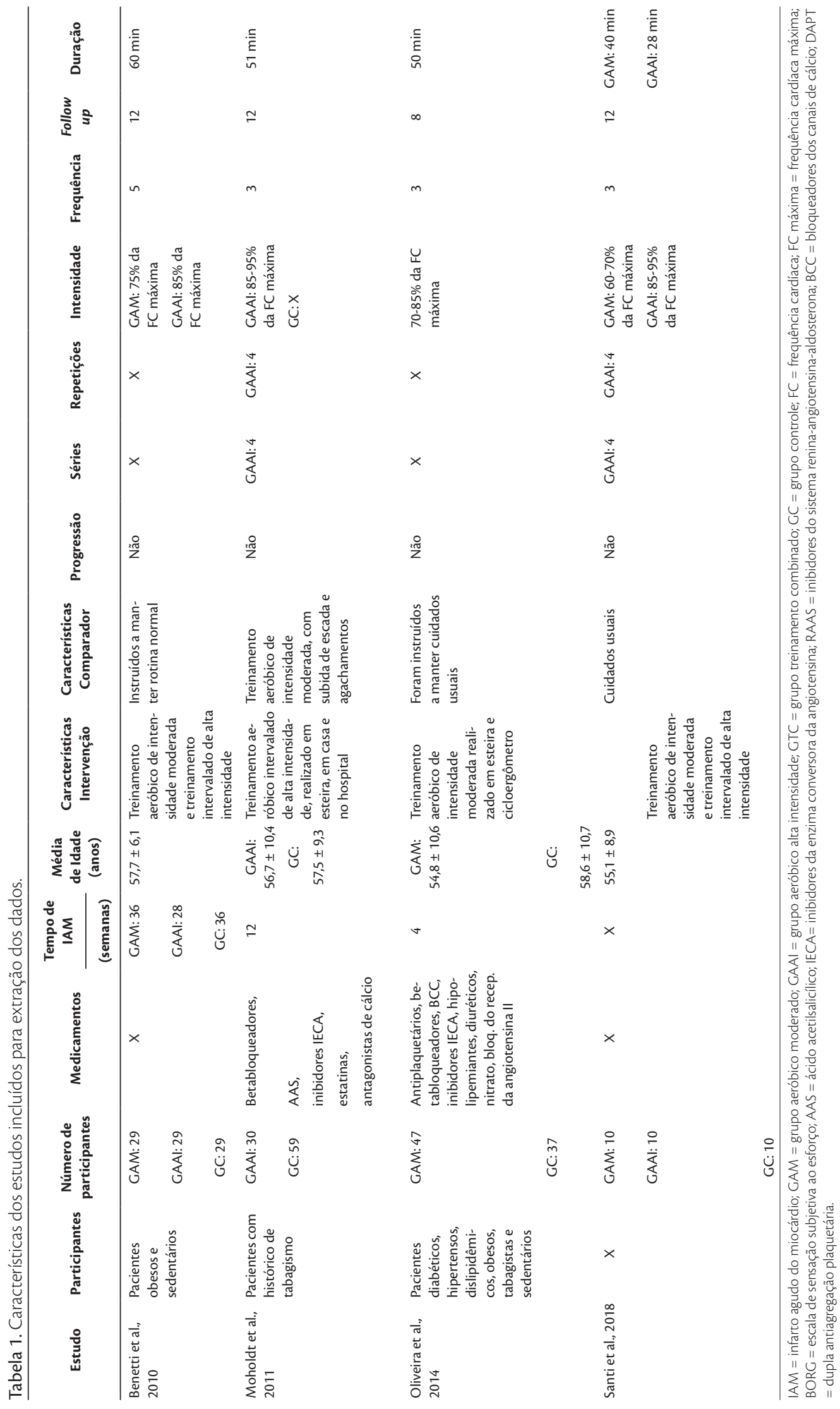




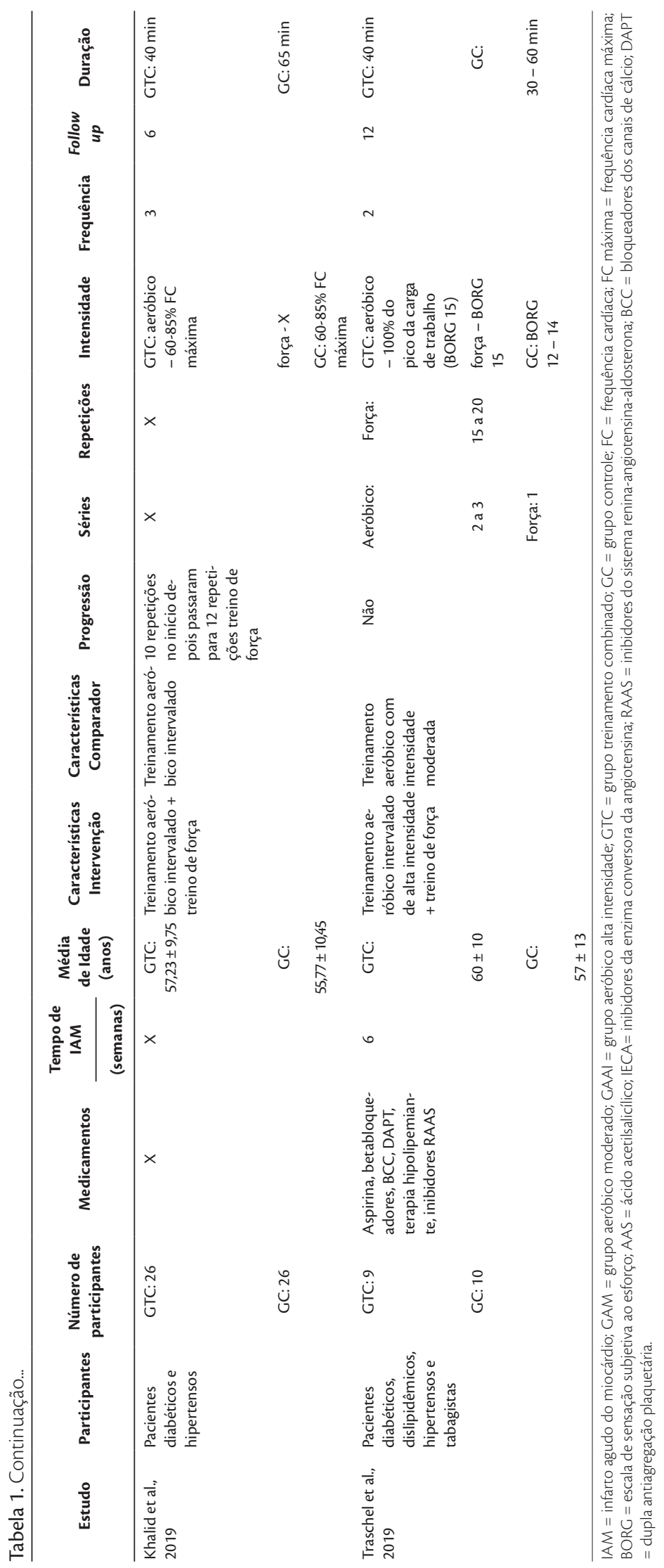


Tabela 2. Extração do risco de viés.

\begin{tabular}{|c|c|c|c|c|c|c|}
\hline Estudo & $\begin{array}{l}\text { Geração da sequência } \\
\text { de randomização }\end{array}$ & $\begin{array}{l}\text { Sigilo de } \\
\text { alocação }\end{array}$ & $\begin{array}{l}\text { Cegamento } \\
\text { paciente e } \\
\text { terapeuta }\end{array}$ & $\begin{array}{l}\text { Cegamento } \\
\text { avaliadores } \\
\text { desfechos }\end{array}$ & $\begin{array}{c}\text { Descrição } \\
\text { perdas e } \\
\text { exclusões }\end{array}$ & $\begin{array}{c}\text { Dados de } \\
\text { resultados } \\
\text { incompletos }\end{array}$ \\
\hline $\begin{array}{l}\text { Benetti et al., } \\
2010\end{array}$ & Baixo & Unclear & Unclear & Unclear & Baixo & Unclear \\
\hline $\begin{array}{l}\text { Mohol- } \\
\text { dt et al., } \\
2011\end{array}$ & Baixo & Baixo & Baixo & Baixo & Baixo & Baixo \\
\hline $\begin{array}{l}\text { Olivei- } \\
\text { ra et al., } \\
2014\end{array}$ & Baixo & Baixo & Alto & Baixo & Baixo & Baixo \\
\hline $\begin{array}{l}\text { Santi et al., } \\
2018\end{array}$ & Baixo & Unclear & Unclear & Unclear & Unclear & Unclear \\
\hline $\begin{array}{l}\text { Khalid et al., } \\
2019\end{array}$ & Baixo & Unclear & Alto & Baixo & Baixo & Alto \\
\hline $\begin{array}{l}\text { Tras- } \\
\text { chel et al., } \\
2019\end{array}$ & Baixo & Unclear & Alto & Baixo & Alto & Unclear \\
\hline
\end{tabular}

Baixo = apresentou o critério, considerado baixo risco de viés; Alto = não apresentou o critério; Unclear = não está claro se possui ou não o critério.

\begin{tabular}{|c|c|}
\hline Estudos & Estimativa (IC95\%) \\
\hline Khalid et al. A 2019 & $1.520(-2.573, \quad 5.613)$ \\
\hline Trachsel et al. A 2019 & $2.900(-4.458,10.258)$ \\
\hline 48) & 1.846 \\
\hline
\end{tabular}

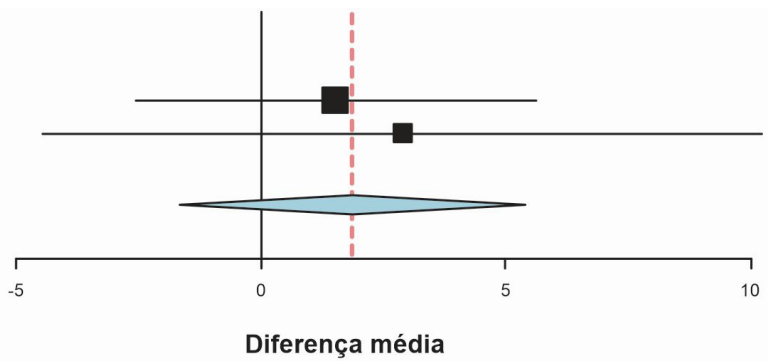

Figura 3. Diferenças médias padronizadas no consumo de oxigênio de pico $\left(\mathrm{mL}^{\mathrm{k}} \mathrm{kg}^{-1} \cdot \mathrm{min}^{-1}\right)$ observadas entre exercício combinado e grupo controle.

Nota: Quadrado preenchido = estimativa dos estudos específicos; Diamante preenchido = estimativas combinadas de metanálises de efeitos aleatórios; Diferença padronizada; Intervalo de confiança.

Apesar da presente revisão sistemática investigar os efeitos de diferentes tipos de treinamento físico, é importante destacar a escassez de estudos que investigam os efeitos do exercício combinado sobre o VO2pico em indivíduos que sofreram IAM $^{31,34}$ e a inexistência de estudos que investigam os efeitos do exercício de força sobre o VO2pico. Em contrapartida, o exercício aeróbico foi a modalidade mais investigada nos estudos $(66 \%)^{30,32,33,35}$. Além disso, o exercício aeróbico de alta intensidade foi utilizado como GC nos dois estudos sobre exercício combinado. Portanto, nossos achados corroboram a literatura que indica o exercício aeróbico como a modalidade mais utilizada para reabilitação de indivíduos que sofreram $\operatorname{IAM}^{19,20}$.

Dos 361 participantes incluídos na presente metanálise, 296 eram do sexo masculino, dado que reforça a prevalência de IAM em indivíduos do sexo masculino ${ }^{4}$. Assim, é importante destacar que um dos fatores de risco para desenvolvimento da doença é o sexo, além da faixa etária ${ }^{5}$. O principal aspecto fisiológico de proteção entre o sexo feminino é o estrógeno, hormônio diretamente relacionado à proteção cardiovascular ${ }^{36}$. Além disso, o IAM afeta pessoas a partir dos 40-45 anos. Nessa faixa etária, os indivíduos do sexo feminino têm sua produção de estrogênio preservada. A maior incidência de IAM no sexo feminino é após a menopausa, quando ocorre uma importante redução na produção de estrogênio ${ }^{36}$.

$\mathrm{O}$ exercício aeróbico tem se mostrado eficaz na redução dos fatores de risco, assim como no aumento da capacidade funcional em indivíduos de meia idade ou idosos nas diferentes DCVs ${ }^{22,37}$. Programas de treinamento físico que contemplem o exercício aeróbico são importantes para promover adaptações positivas no VO2pico em indivíduos que sofreram IAM. Entretanto, é fundamental o manejo da intensidade de exercício para que se obtenha os resultados esperados durante uma intervenção ${ }^{38}$. $\mathrm{O}$ exercício aeróbico com alta intensidade parece ser o que apresenta maior magnitude de aumento no 
VO2pico $\left(14,7 \mathrm{~mL} \cdot \mathrm{kg}^{-1} \cdot \mathrm{min}^{-1}\right)^{30}$. A alta intensidade, 85-95\% do VO2pico, além de apresentar boa aderência em programas de intervenção com exercícios físicos, apresenta aumento na capacidade oxidativa dos músculos e capacidade de transporte de glicose, consequentemente melhorando a sensibilidade à insulina e o controle glicêmico $^{39}$. O principal mecanismo de adaptação em função da alta intensidade está relacionado ao receptor-y coativador- $\alpha$ de peroxissoma, o mais importante regulador da biogênese mitocondrial no músculo ${ }^{40}$.

O mecanismo regulador do exercício aeróbico de moderada intensidade parece ser semelhante. O aspecto central é a biogênese mitocondrial, importante organela celular responsável pela atividade oxidativa dos músculos ${ }^{41,42}$. Aparentemente, seis 6 semanas de exercício aeróbico de moderada intensidade são necessárias para promover aumento tanto no tamanho quanto no número de mitocôndrias, gerando maior capacidade de ressíntese de adenosina trifosfato. Esse dado corrobora um dos critérios de inclusão do presente estudo, visto que foi determinado um tempo mínimo de 6 semanas de intervenção ${ }^{43}$. Além disso, o exercício aeróbico de modo geral aumenta a diferença arteriovenosa de oxigênio, a qual está diretamente relacionada ao aumento no VO2pico, por meio de uma maior oferta de oxigênio periférico, em função da produção de catecolaminas e maior biodisponibilidade de óxido nítrico ${ }^{44,45}$. A função cardíaca também apresenta maior desempenho após uma intervenção com exercício aeróbico, quando ocorre um aumento no enchimento diastólico, com uma concomitante combinação de pré-carga aumentada e relaxamento do miocárdio otimizado ${ }^{46}$. A melhora na função cardíaca promoverá importantes benefícios para a redução no tempo de diástole e maior eficiência no enchimento diastólico ${ }^{46}$.

Do ponto de vista clínico, o principal achado do presente estudo corrobora a literatura sobre o papel da reabilitação por meio de exercícios aeróbicos em indivíduos que sofreram IAM, utilizando como preferência a moderada intensidade ${ }^{47}$, em virtude de as diferentes diretrizes possuírem recomendação I com nível de evidência A, ou seja, altamente indicados para diferentes $\mathrm{DCVs}^{19-21}$. Um aumento médio de $6 \mathrm{~mL} \cdot \mathrm{kg}^{-1} \cdot \mathrm{min}^{-1}$ no VO2pico, achado principal deste estudo, demonstra que o exercício físico exerce papel fundamental na redução do risco de mortalidade por DCVs, uma vez que o aumento de uma unidade no VO2pico representa redução do risco de mortalidade por DCVs de $10 \%{ }^{16,17}$. Além disso, uma diferença de 6 $\mathrm{mL} \cdot \mathrm{kg}^{-1} \cdot \mathrm{min}^{-1}$ pode aumentar a autonomia do indivíduo nas suas atividades diárias, visto que essa diferença pode representar uma modificação na classe funcional, tanto do New York Heart Association (NYHA) quanto Weber, quando, por exemplo, um indivíduo classe III do NYHA e classe C de Weber pode mudar para classe I do NYHA e classe A de Weber ${ }^{47,48}$. Portanto, o achado deste estudo é extremamente relevante para a área da reabilitação cardiovascular.

Apesar do exercício combinado não apresentar diferenças significativas em comparação a um GC $(\mathrm{p}=0,312)$, é preciso destacar a inclusão de apenas dois estudos para esse tipo de intervenção, o que pode ser extremamente relevante para não encontrar significância. A diferença média de aumento no VO2pico para o exercício combinado em comparação ao $\mathrm{GC}$ foi de $1,84 \mathrm{~mL} \cdot \mathrm{kg}^{-1} \cdot \mathrm{min}^{-1}$. A combinação entre exercício aeróbico e exercício de força na mesma sessão parece ser uma estratégia promissora - além de promover melhora no VO2pico, diferentes estudos apontam a importância do exercício combinado para a melhora de aspectos neuromusculares relacionados ao equilíbrio e força muscular, principalmente por meio da realização de exercício de força ${ }^{23,29}$. Ademais, o exercício de força promove aumento no tamanho das fibras musculares, com consequente aumento no número de mitocôndrias, facilitando a atividade oxidativa muscular ${ }^{49}$. Por isso, é importante que novas investigações abordem diferentes programas de treinamento físico sobre diferentes desfechos em indivíduos que sofreram IAM.

Não podemos deixar de destacar a extensa busca na literatura por artigos científicos com alta qualidade metodológica e melhor nível de evidência disponível. Mais de 4.000 estudos foram selecionados para revisão sistemática, com a leitura de títulos e resumos. Entendemos que essa é a primeira metanálise a investigar os efeitos de diferentes modalidades de treinamento físico sobre o VO2pico em indivíduos que sofreram IAM.

\section{CONCLUSÃO}

Nossos dados demonstraram que os participantes que foram treinados com a modalidade exercício aeróbico obtiveram um aumento significativo no VO2pico. Além disso, é a modalidade mais utilizada nos estudos incluídos na presente metanálise. O exercício combinado não apresenta um aumento significativo no VO2pico, mas sua utilização em um programa de reabilitação cardiovascular é de extrema importância, visto que indivíduos que sofreram IAM, além de apresentarem redução em sua capacidade funcional, apresentam perda de força muscular e redução no equilíbrio. Portanto, é fundamental incentivar a realização de programas de treinamento físico para população que sofreu IAM, com adequado controle de intensidade e volume de exercício. 


\section{REFERÊNCIAS}

1. Roth $\mathrm{GA}$, Johnson C, Abajobir A, et al. Global, regional, and national burden of cardiovascular diseases for 10 causes, 1990 to 2015. J Am Coll Cardiol. 2017;70(1):1-25. http://dx.doi.org/10.1016/j. jacc.2017.04.052. PMid:28527533.

2. Martins WA, Rosa MLG, Matos RC, et al. Trends in mortality rates from cardiovascular disease and cancer between 2000 and 2015 in the most populous capital cities of the five regions of Brazil. Arq Bras Cardiol. 2020;114(2):199-206. PMid:32215484.

3. Oliveira GMM, Brant LCC, Polanczyk CA, et al. Estatística cardiovascular Brasil 2020. Arq Bras Cardiol. 2020;115(3):308-439. http://dx.doi.org/10.36660/abc.20200812.

4. Ribeiro AL, Duncan BB, Brant LC, Lotufo PA, Mill JG, Barreto SM. Cardiovascular health in Brazil: trends and perspectives. Circulation. 2016;133(4):422-33. http://dx.doi.org/10.1161/ CIRCULATIONAHA.114.008727. PMid:26811272.

5. Tibaut $M$, Mekis D, Petrovic D. Pathophysiology of myocardial infarction and acute management strategies. Cardiovasc Hematol Agents Med Chem. 2017;14(3):150-9. http://dx.doi.org/10.2174/ 1871525714666161216100553 . PMid:27993119.

6. Marcolino MS, Brant LCC, Araujo JG, et al. Implementation of the myocardial infarction system of care in city of Belo Horizonte, Brazil. Arq Bras Cardiol. 2013;100:307-14. PMid:23545995.

7. Bertuzzi M, Negri E, Tavani A, Vecchia C. Family history of ischemic heart disease and risk of acute myocardial infarction. Prev Med. 2003;37(3):183-7. http://dx.doi.org/10.1016/S0091-7435(03)00094-X PMid: 12914823.

8. Joshi NV, Toor I, Shah AS, et al. Systemic atherosclerotic inflammation following acute myocardial infarction: myocardial infarction begets myocardial infarction.J Am Heart Assoc. 2015;4(9):e001956. http:// dx.doi.org/10.1161/JAHA.115.001956. PMid:26316523.

9. Crea F, Liuzzo G. Pathogenesis of acute coronary syndromes. J Am Coll Cardiol. 2013;61(1):1-11. http://dx.doi.org/10.1016/j. jacc.2012.07.064. PMid:23158526.

10. Allahverdian S, Pannu PS, Francis GA. Contribution of monocytederived macrophages and smooth muscle cells to arterial foam cell formation. Cardiovasc Res. 2012;95(2):165-72. http://dx.doi. org/10.1093/cvr/cvs094. PMid:22345306.

11. Madsen EB, Gilpin E, Ahnve S, Henning H, Ross J Jr. Prediction of functional capacity and use of exercise testing for predicting risk after acute myocardial infarction. Am J Cardiol. 1985;56(13):839-45. http://dx.doi.org/10.1016/0002-9149(85)90766-0. PMid:2865888.

12. Kunz VC, Serra KBS, Borges ÉN, Serra PES, Silva E. Cardiopulmonary exercise testing in the early-phase of myocardial infarction. Rev Bras Fisioter. 2012;16(5):396-405. http://dx.doi.org/10.1590/ S1413-35552012005000047. PMid:23032293.

13. Chiaranda G, Myers J, Arena R, et al. Improved percent-predicted peak $\mathrm{VO}(2)$ is associated with lower risk of hospitalization in patients with coronary heart disease. Analysis from the FRIEND registry. Int J Cardiol. 2020;310:138-44. http://dx.doi.org/10.1016/j. ijcard.2020.02.057. PMid:32139240.

14. Siqueira CAS, Souza DLB. Reduction of mortality and predictions for acute myocardial infarction, stroke, and heart failure in Brazil until 2030. Sci Rep. 2020;10(1):17856.

15. Swank AM, Horton J, Fleg JL, et al. Modest increase in peak VO2 is related to better clinical outcomes in chronic heart failure patients: results from heart failure and a controlled trial to investigate outcomes of exercise training. Circ Heart Fail. 2012;5(5):579-85. http://dx.doi.org/10.1161/CIRCHEARTFAILURE.111.965186. PMid:22773109.
16. Kavanagh T, Mertens DJ, Hamm LF, et al. Prediction of long-term prognosis in 12169 men referred for cardiac rehabilitation. Circulation. 2002;106(6):666-71. http://dx.doi.org/10.1161/01. CIR.0000024413.15949.ED. PMid:12163425.

17. Kavanagh T, Mertens DJ, Hamm LF, et al. Peak oxygen intake and cardiac mortality in women referred for cardiac rehabilitation. J Am Coll Cardiol. 2003;42(12):2139-43. http://dx.doi.org/10.1016/j. jacc.2003.07.028. PMid:14680741.

18. Anderson L, Sharp GA, Norton RJ, et al. Home-based versus centre-based cardiac rehabilitation. Cochrane Database Syst Rev. 2017;6(6):CD007130. PMid:28665511.

19. Pelliccia A, Sharma S, Gati S, et al. 2020 ESC Guidelines on sports cardiology and exercise in patients with cardiovascular disease. Eur Heart J. 2021;42(2):17-96. PMid:32860412.

20. Leon AS, Franklin BA, Costa F, et al. Cardiac rehabilitation and secondary prevention of coronary heart disease: an American Heart Association scientific statement from the Council on Clinical Cardiology (Subcommittee on Exercise, Cardiac Rehabilitation, and Prevention) and the Council on Nutrition, Physical Activity, and Metabolism (Subcommittee on Physical Activity), in collaboration with the American association of Cardiovascular and Pulmonary Rehabilitation. Circulation. 2005;111(3):369-76. http://dx.doi. org/10.1161/01.CIR.0000151788.08740.5C. PMid:15668354.

21. Carvalho T, Milani M, Ferraz AS, et al. Brazilian cardiovascular rehabilitation guideline - 2020. Arq Bras Cardiol. 2020;114(5):94387. http://dx.doi.org/10.36660/abc.20200407. PMid:32491079.

22. Peixoto TC, Begot I, Bolzan DW, et al. Early exercise-based rehabilitation improves health-related quality of life and functional capacity after acute myocardial infarction: a randomized controlled trial. Can J Cardiol. 2015;31(3):308-13. http://dx.doi.org/10.1016/j. cjca.2014.11.014. PMid:25633911.

23. Pierson LM, Herbert WG, Norton HJ, et al. Effects of combined aerobic and resistance training versus aerobic training alone in cardiac rehabilitation.J Cardiopulm Rehabil. 2001;21(2):101-10. http:// dx.doi.org/10.1097/00008483-200103000-00007. PMid:11314283.

24. Meka N, Katragadda S, Cherian B, Arora RR. Endurance exercise and resistance training in cardiovascular disease. Ther Adv Cardiovasc Dis. 2008;2(2):115-21. http://dx.doi.org/10.1177/1753944708089701. PMid:19124415.

25. Higgins JPT, Deeks JJ, Altman DG. Cochrane handbook for systematic reviews of interventions, version 5.1. Oxford: Cochrane Collaboration; 2011.

26. Moher D, Liberati A, Tetzlaff J, Altman DG. Preferred reporting items for systematic reviews and meta-analyses: the PRISMA Statement. Open Med. 2009;3(3):e123-30. PMid:21603045.

27. Higgins JP, Thompson SG, Deeks JJ, Altman DG. Measuring inconsistency in meta-analyses. BMJ. 2003;327(7414):557-60. http://dx.doi.org/10.1136/bmj.327.7414.557. PMid:12958120.

28. Takagi S, Murase N, Kime R, Niwayama M, Osada T, Katsumura T. Aerobic training enhances muscle deoxygenation in early postmyocardial infarction. Eur J Appl Physiol. 2016;116(4):673-85. http://dx.doi.org/10.1007/s00421-016-3326-x. PMid:26759155.

29. Arthur HM, Gunn E, Thorpe KE, et al. Effect of aerobic vs combined aerobic-strength training on 1-year, post-cardiac rehabilitation outcomes in women after a cardiac event. J Rehabil Med. 2007;39(9):730-5. http://dx.doi.org/10.2340/16501977-0122. PMid:17999012.

30. Benetti M, Araujo CL, Santos RZ. Cardiorespiratory fitness and quality of life at different exercise intensities after myocardial infarction. Arq Bras Cardiol. 2010;95(3):399-404. http://dx.doi. org/10.1590/S0066-782X2010005000089. PMid:20640381. 
31. Khalid Z, Farheen $\mathrm{H}$, Tariq MI, Amjad I. Effectiveness of resistance interval training versus aerobic interval training on peak oxygen uptake in patients with myocardial infarction. J Pak Med Assoc. 2019;69(8):1194-8. PMid:31431779.

32. Santi GLD, Moreira HT, Carvalho EEV, et al. Influence of aerobic training on the mechanics of ventricular contraction after acute myocardial infarction: a pilot study. Arq Bras Cardiol. 2018;110(4):3837. http://dx.doi.org/10.5935/abc.20180049. PMid:29791580.

33. Oliveira NL, Ribeiro F, Teixeira M, et al. Effect of 8-week exercisebased cardiac rehabilitation on cardiac autonomic function: a randomized controlled trial in myocardial infarction patients. Am Heart J. 2014;167(5):753-61. http://dx.doi.org/10.1016/j. ahj.2014.02.001. PMid:24766987.

34. Trachsel LD, David LP, Gayda M, et al. The impact of high-intensity interval training on ventricular remodeling in patients with a recent acute myocardial infarction - a randomized training intervention pilot study. Clin Cardiol. 2019;42(12):1222-31. http://dx.doi. org/10.1002/clc.23277.

35. Moholdt T, Aamot IL, Granøien I, et al. Aerobic interval training increases peak oxygen uptake more than usual care exercise training in myocardial infarction patients: a randomized controlled study. Clin Rehabil. 2012;26(1):33-44. http://dx.doi. org/10.1177/0269215511405229. PMid:21937520.

36. lorga A, Cunningham CM, Moazeni S, Ruffenach G, Umar S, Eghbali $M$. The protective role of estrogen and estrogen receptors in cardiovascular disease and the controversial use of estrogen therapy. Biol Sex Differ. 2017;8(1):33. http://dx.doi.org/10.1186/ s13293-017-0152-8. PMid:29065927.

37. Franzoni L, Stein R. Moderate exercise improves depressive symptoms and pain in elderly people. Int J Cardiovasc Sci. 2019;32(6):563-4.

38. Stein R, Franzoni LT. Digital tools and cardiovascular rehabilitation. Int J Cardiovasc Sci. 2018;31(6):558-9.

39. Liu JX, Zhu L, Li PJ, Li N, Xu YB. Effectiveness of high-intensity interval training on glycemic control and cardiorespiratory fitness in patients with type 2 diabetes: a systematic review and metaanalysis. Aging Clin Exp Res. 2019;31(5):575-93. http://dx.doi. org/10.1007/s40520-018-1012-z. PMid:30097811.

40. Li J, Li Y, Atakan MM. The molecular adaptive responses of skeletal muscle to high-intensity exercise/training and hypoxia. Antioxidants. 2020;9(8):656.

41. Menshikova EV, Ritov VB, Fairfull L, Ferrell RE, Kelley DE, Goodpaster $\mathrm{BH}$. Effects of exercise on mitochondrial content and function in aging human skeletal muscle. J Gerontol A Biol Sci Med Sci. 2006;61(6):534-40. http://dx.doi.org/10.1093/gerona/61.6.534. PMid:16799133.

42. Distefano G, Goodpaster BH. Effects of exercise and aging on skeletal muscle. Cold Spring Harb Perspect Med. 2018;8(3):a029785. http://dx.doi.org/10.1101/cshperspect.a029785. PMid:28432116.

43. Clark JE. The impact of duration on effectiveness of exercise, the implication for periodization of training and goal setting for individuals who are overfat, a meta-analysis. Biol Sport. 2016;33(4):309-33. http://dx.doi.org/10.5604/20831862.1212974. PMid:28090136.

44. Detry JM, Rousseau M, Vandenbroucke G, Kusumi F, Brasseur LA, Bruce RA. Increased arteriovenous oxygen difference after physical training in coronary heart disease. Circulation. 1971;44(1):109-18. http://dx.doi.org/10.1161/01.CIR.44.1.109. PMid:5561413.

45. Di Francesco Marino S, Sciartilli A, Di Valerio V, Di Baldassarre A, Gallina $S$. The effect of physical exercise on endothelial function. Sports Med. 2009;39(10):797-812. http://dx.doi.org/10.2165/11317750000000000-00000. PMid:19757859.
46. Garcia EL, Menezes MG, Stefani CM, Danzmann LC, Torres MA. Ergospirometry and echocardiography in early stage of heart failure with preserved ejection fraction and in healthy individuals. Arq Bras Cardiol. 2015;105(3):248-55. PMid:26247247.

47. Arena R, Sietsema KE. Cardiopulmonary exercise testing in the clinical evaluation of patients with heart and lung disease. Circulation. 2011;123(6):668-80. http://dx.doi.org/10.1161/ CIRCULATIONAHA.109.914788. PMid:21321183.

48. Belli KC, Silva PFD, Franzoni LT, Myers J, Stein R, Ribeiro JP. Speed and grade increment during cardiopulmonary treadmill testing: impact on exercise prescription. Int J Cardiovasc Sci. 2019;32:37483. http://dx.doi.org/10.5935/2359-4802.20190058.

49. Balakrishnan VS, Rao M, Menon V, et al. Resistance training increases muscle mitochondrial biogenesis in patients with chronic kidney disease. Clin J Am Soc Nephrol. 2010;5(6):996-1002. http://dx.doi. org/10.2215/CJN.09141209. PMid:20498251.

Correspondência Eduardo Lima Garcia Hospital de Clínicas de Porto Alegre - HCPA/UFRGS Avenida Professor Oscar Pereira 980 90640-070 - Porto Alegre (RS), Brasil Tel.: (51) 998418989 E-mail: rceduardogarcia@gmail.com

Informações sobre os autores: GB - Fisioterapeuta, Faculdade Cenecista de Santo Ângelo; Clínica de Fisioterapia Equipe Silvana. KRJ - Fisioterapeuta, Faculdade Cenecista de Santo Ângelo. RRC - Doutora em Ciências do Movimento Humano, Universidade Federal do Rio Grande do Sul (UFRGS); Professora Titular, Curso de Educação Física, Faculdade SOGIPA. TP - Formanda em Educação Física, Universidade Federal de Santa Maria (UFSM); área de Reabilitação Cardíaca, Hospital de Santa Maria.

LCD - Professor Titular, Curso de Medicina, Universidade Luterana do Brasil (ULBRA); Chefe, Ambulatório de Insuficiência Cardíaca, Hospital Universitário, ULBRA.

AHP - Chefe do Ambulatório de Cirurgia Vascular do Hospital de Clínicas de Porto Alegre (HCPA/UFRGS); Professor Titular da Faculdade de Medicina da Universidade Federal do Rio Grande do Sul (UFRGS). AAP - Cirurgião Vascular, Ambulatório de Cirurgia Vascular, Hospital de Clínicas de Porto Alegre (HCPA/UFRGS); Doutorando, Programa

de Ciências Médicas, Universidade Federal do Rio Grande do Sul (UFRGS).

LTF - Doutorando, Cardiologia e Ciências Cardiovasculares, Universidade Federal do Rio Grande do Sul (UFRGS); Membro do Grupo de Pesquisa VascuEx, Ambulatório de Cirurgia Vascular, Hospital de Clínicas de Porto Alegre (HCPA/UFRGS).

ELG - Doutor em Medicina; Coordenador, Grupo de Pesquisa VascuEx, Ambulatório de Cirurgia Vascular, Hospital de Clínicas de Porto Alegre (HCPA/UFRGS).

Contribuição dos autores: Concepção e desenho do estudo: GB, KRJ Análise e interpretação dos dados: LTF, ELG Coleta de dados: GB, KRJ, TP Redação do artigo: LTF, ELG, LCD, AHP Revisão crítica do texto: AHP, AAP, LCD, ELG Aprovação final do artigo*: AHP, RRC, AAP, ELG Análise estatística: LTF, RRC

Responsabilidade geral pelo estudo: ELG

*Todos os autores leram e aprovaram a versão final submetida do J Vasc Bras. 